\title{
Lack of NOD2 attenuates ovariectomy-induced bone loss via inhibition of osteoclasts
}

\author{
Ke Ke ${ }^{1, *}$, Ok-Joo Sul1,*, Soo-Wol Chung', Jae-Hee Suh² and Hye-Seon Choi ${ }^{1}$ \\ 1Department of Biological Sciences, University of Ulsan, Ulsan, Korea \\ 2Department of Pathology, Ulsan University Hospital, Ulsan, Korea \\ *(K Ke and O-J Sul contributed equally to this work)
}

Correspondence

should be addressed

to H-S Choi

Email

hschoi@mail.ulsan.ac.kr

\begin{abstract}
Nucleotide-binding oligomerization domain-2 (NOD2) is a pattern recognition receptor of the innate immune system. It interacts with serine-threonine kinases to induce activation of nuclear factor $\mathrm{KB}$ (NF-kB), which is important for receptor activator of nuclear factor kappa-B ligand (RANKL) signaling. We tested the idea that NOD2 modulates bone metabolism via an action on osteoclasts (OCs). The absence of NOD2 reduced ovariectomy-induced bone loss in mice, and lowered the area and the activity of OCs, by impairing RANKL signaling. It also reduced the level of reactive oxygen species (ROS), as well as of NF-KB-DNA binding upon RANKL exposure. NOD2 was found to physically interact with nicotinamide adenine dinucleotide phosphate oxidase 1 , and this led to increased production of ROS in OCs. Our data suggest that NOD2 contributes to bone loss in estrogen deficiency by elevating ROS levels in OCs.
\end{abstract}

\author{
Key Words \\ - nucleotide-binding \\ oligomerization domain 2 \\ - osteoclast \\ - receptor activator of \\ nuclear factor-kB ligand \\ signaling \\ - reactive oxygen species \\ - nicotinamide adenine \\ dinucleotide phosphate \\ oxidase 1
}

\section{Introduction}

Bone is a dynamic tissue, aging bone being constantly replaced by new tissue. Bone remodeling occurs via the balanced action of osteoblasts and OCs. Loss of ovarian function increases the rate of bone remodeling and results in a loss of bone mass due to excessive bone resorption (Weitzmann \& Pacifici 2006). The enhanced bone remodeling enlarges the remodeling space and increases cortical porosity and the resorption area on trabecular surfaces (Eriksen et al. 1999). This is primarily due to elevated osteoclastogenesis involving neighboring cells (Weitzmann \& Pacifici 2006), as well as increased resorption due to longer OC survival (Hughes et al. 1996). Since recent findings suggest a close interrelationship between bone and immune system, postmenopausal osteoporosis could be an example reflecting mutual influence of immune system, bone and endocrine system (Ginaldi \& De Martinis 2016). Ovariectomy (OVX) in mice is a commonly accepted model reflecting the human menopause. Loss of ovarian function has been reported to be associated with metabolic pathologies mainly due to chronic inflammation (Rogers et al. 2009, Choi et al. 2013, 2015, Kim et al. 2013), suggesting that chronic inflammation contributes to the metabolic complications such as bone loss observed upon OVX.

OCs, multinucleated giant cells responsible for bone resorption, are formed from hematopoietic cells 
of the monocyte/macrophage lineage and share some of their morphological and functional properties. They are responsible not only for physiological bone remodeling, but also for the bone destruction associated with chronic inflammatory disease (Crotti et al. 2015, Ginaldi \& De Martinis 2016). However, OCs are affected by other OCs as well as by cross-talk with neighboring cells. To promote osteoclastogenesis, two essential molecules are generated by bone marrow mesenchymal cells: macrophage-colony stimulating factor (M-CSF) and RANKL, a member of the tumor necrosis factor (TNF) family (Kong et al. 1999, Suda et al. 1999). Binding of RANKL to its receptor, RANK, on OC precursor cells induces and activates transcription factors including NF-кB (Cappellen et al. 2002) needed to initiate signals for OC differentiation, and this results in the expression of OC-specific genes such as tartrateresistant acid phosphatase (TRAP) and calcitonin receptor.

Different pathogen-associated molecular patterns (PAMPs) such as flagellin, which are important microbial components, unique nucleic acid structures and bacterial cell wall constituents, are recognized by the corresponding specific receptors. Toll-like receptors (TLR) sense PAMPs on the cell surface and within endosomes, whereas a cytosolic pattern recognition receptor (PRR), nucleotidebinding and oligomerization domain 2 (NOD2) recognizes muramyldipeptides (MDPs), breakdown products of bacterial cell walls in the cytosol (Girardin et al. 2003), suggesting another way that invading microorganisms can be recognized. Activation of NOD2 is triggered by direct or indirect recognition of MDPs through its C-terminal leucine-rich repeat region (LRR) in the cytosol, and is followed by NOD-mediated oligomerization and direct interaction with receptor-interacting protein 2, leading to the formation of a NOD2 'signalosome complex' and further activation of NF-kB (Rosenstiel et al. 2008). Mutation of NOD2, which is involved in susceptibility to Crohn's disease, a chronic inflammatory intestinal disorder, results in impaired MDP recognition and attenuated NF-кB activation (Hugot et al. 2001), indicating that NOD2 is involved in many aspects of chronic inflammation. In the present study, we investigated the hypothesis that NOD2 promotes OVX-induced bone loss by amplifying RANKL-induced signaling in OCs.

\section{Materials and methods}

\section{Animals and study design}

Nod2-I- (Nod2-knockout (KO)) mice were purchased from the Jackson Laboratory. The mice were bred with C57BL/6J mice and maintained by crossing in the animal facility of the University of Ulsan. Six-week-old Nod2+/+ (WT) and $\mathrm{Nod2}^{-I-}$ (Nod2-KO) mice on a background of C57BL/6J mice were subjected to OVX $(n=6)$ or sham operation $(n=6)$ under anesthesia using a mixture of Zoletil and Rompun. Eight weeks after surgery, bone density was analyzed, and genomic DNA was extracted from the tails of the mice for genotyping by PCR. All mice were housed in a specific pathogen-free animal facility, and animal care and all procedures were conducted according to protocols and guidelines approved by the University of Ulsan Animal Care and Use Committee (UOUACUC). The standards employed were approved by the latter Committee (UOU2011-009). For visualizing the architecture of the long bone, femurs were analyzed by scanning with a highresolution micro-computed tomography $(\mu \mathrm{CT})$ imaging system in a SkyScan 1072 System (SkyScan, Kontich, Belgium) set to $6.9 \mu \mathrm{m}$ effective detector pixel size and a threshold of $77-255 \mathrm{mg} / \mathrm{cc}$. Trabecular bone was assessed in a $1.5 \mathrm{~mm}$ region $0.2 \mathrm{~mm}$ below the distal growth plate of the femur. Three-dimensional analyses were performed with CT volume software (ver. 1.11; SkyScan) using a total of 250-300 tomographic slices. To examine in vivo TRAP-positive OCs, mouse femora were excised, cleaned with a soft tissue and decalcified in EDTA. Representative histological sections of the distal femoral metaphyses of WT and Nod2-KO mice were stained for TRAP to identify OCs (original magnification, $\times 200$ ). As in vivo markers of bone resorption, serum C-telopeptide fragments of collagen type 1 (CTX-1) were determined with a RatLaps enzyme immunoassay (EIA) according to the manufacturer's instructions (Immunodiagnostic Systems Inc., Woburn, MA, USA). Serum osteocalcin was measured with an osteocalcin EIA kit (Biomedical Technologies Inc., Stoughton, MA, USA) and alkaline phosphatase (ALP) by a colorimetric kinetic assay (BioAssay Systems, Hayward, CA, USA). Serum $\mathrm{H}_{2} \mathrm{O}_{2}$ was evaluated with an OxiSelect hydrogen peroxide assay kit (Cell Biolabs, Inc., San Diego, CA, USA).

\section{OC formation}

Bone marrow cells were isolated from mice as described previously (Van Phan et al. 2013). Cells from sham and OVX mice were obtained for ex vivo OC formation 7-8 weeks after surgery. Femora and tibiae were removed aseptically and dissected free of adherent soft tissue. The marrow cavity was washed with $\alpha$-MEM from one end of the bone using a sterile 21-gauge needle after dissecting the bone ends, and a single-cell suspension was prepared with

Published by Bioscientifica Ltd 
a Pasteur pipette. The resulting bone marrow suspension was washed twice and incubated on plates with M-CSF $(20 \mathrm{ng} / \mathrm{mL})$ for $16 \mathrm{~h}$. Floating cells were harvested and loaded on a Ficoll-Hypaque gradient and the cells at the interface were harvested. Two more days of incubation resulted in large populations of monocyte/macrophagelike cells adhering to the culture plates. Floating cells were discarded by washing the dishes with phosphate-buffered saline (PBS), and the adherent cells (bone marrow-derived macrophages (BMMs)) were collected and seeded on plates. These cells were analyzed with a FACSCalibur flow cytometer (Becton Dickinson, Franklin Lakes, NJ, USA) and found to be negative for CD3 and CD45R and positive for CD11b (Ke et al. 2015). The lack of growth without M-CSF confirmed the absence of contaminating stromal cells. M-CSF and RANKL ( $40 \mathrm{ng} / \mathrm{mL}$ ) were added to the cells, and the incubation medium was replaced on day 3. When MDP $(10 \mu \mathrm{g} / \mathrm{mL}$, Sigma Chemical) was treated, it was added to the cells $10 \mathrm{~min}$ before RANKL treatment. After incubation for the indicated times, the cells were fixed in 10\% formalin for $10 \mathrm{~min}$ and stained for TRAP as described (Van Phan et al. 2013). OC numbers were evaluated blind by counting TRAP-positive multinucleated cells (MNCs) (three or more nuclei) per well using an eye piece graticule at a magnification of $\times 100$. Cultures in the presence of M-CSF with and without RANKL were used as positive and negative control, respectively. Whole bone marrow cells from sham and OVX mice were stimulated with $1,25(\mathrm{OH})_{2} \mathrm{D}_{3}(10 \mathrm{nM})$ and $\mathrm{PGE}_{2}(1 \mu \mathrm{M})$ for ex vivo OC formation for 7-8 days, and the incubation medium was replaced every 3 days. For $\mathrm{E}_{2}$ treatment in vitro, BMMs were cultured in $\alpha$-MEM without phenol red (Life Technologies) containing 10\% charcoaltreated FBS (de Faria et al. 2016). The resorptive activity of OCs was determined with a lacunar resorption assay on dentine disks placed in wells (Jimi et al. 1999). Mature OC cells were generated by incubation with M-CSF and RANKL in the presence or absence of MDP on dentine slices for 6-7 days. Mature OCs with and without RANKL in the presence of M-CSF were used as positive and negative controls, respectively. The slices were cleaned by ultrasonication in $1 \mathrm{M} \mathrm{NH}_{4} \mathrm{OH}$ to remove adherent cells and stained with Mayer's hematoxylin (Sigma) to visualize resorption pits. Resorption pit areas were measured with Image $1.37 \mathrm{v}$.

\section{Transfection of BMMs with siRNA}

The BMMs were transfected with small interfering RNAs (siRNA) against Nod2 (sc-30199; Santa Cruz
Biotechnology), Nox1 (sc-25545) or scrambled siRNA (scRNA) (Santa Cruz) using Lipofectamine RNAiMAX (Invitrogen). Lipofectamine diluted in $\alpha$-MEM was mixed with an equal volume of $\alpha$-MEM containing the siRNA. After $20 \mathrm{~min}$ of incubation, $100 \mu \mathrm{L}$ of RNAiMAX/siRNA were added to the cells, to a final volume of $700 \mu \mathrm{L}$. After 8-h incubation, the cells were replated in serumcontaining medium, cultured for another 2 days, and mRNA levels were analyzed by quantitative polymerase chain reaction (qPCR).

\section{RNA isolation and qPCR}

Total RNA was reverse-transcribed with random primers and M-MLV reverse transcriptase (Promega). qPCR used SYBR Green 1 Taq polymerase (Qiagen) and appropriate primers on a StepOnePlus Real-Time System (Applied Biosystems). Primer specificity was confirmed by melting curve analysis and agarose-gel electrophoresis. The housekeeping gene for GAPDH was amplified in parallel with genes of interest. Relative copy numbers compared to GAPDH were calculated using 2- $2^{-\Delta C t}$. Primer sequences were $5^{\prime}$-ggcaccctgaagttgacattttgc- $3^{\prime}$ and $\quad 5^{\prime}$-acatctcccacagagttgtaatcc-3' (NOD2); $\quad 5^{\prime}$-tccatttccttcctggagtggcat-3' and $5^{\prime}$-ggcattggtgagtgctgttgttca-3'

(NOX1);

5'-ctccaacaaggtgcttggga-3' and 5'-gaagcagtagatagtcgcca-3' (calcitonin receptor); 5'-gaccaccttggcaatgtctctg-3' and $\quad 5^{\prime}$-tggctgaggaagtcatctgagttg- $3^{\prime} \quad$ (TRAP); 5'-gtgggtgttcaagtttctgc-3' and 5'-ggtgagtcttcttccatagc-3' (cathepsin K); $5^{\prime}$-aataacatgcgagccatcatc- $3^{\prime}$ and $5^{\prime}$-tcaccctggtgttcttcctc-3' (nuclear factor of activated T cells, cytoplasmic 1 (NFAT2)); 5'-agacgtggtttaggaatgcagctc-3' and $\quad 5^{\prime}$-tcctccatgaacaaacagttccaa-3' (DC-STAMP); $\quad 5^{\prime}$-ttcagttgctatccaggactcgga-3' and $\quad 5^{\prime}$-gcatgtcatgtaggtgagaaatgtgctca-3' (ATP6v0d2) and 5'-acccagaagactgtggatgg-3' and 5'-cacattgggggtaggaacac-3' (GAPDH). Primer sequences for RT-PCR were 5'-atgggaaactggctggttaaccac-3' and $\quad 5^{\prime}$-ggcattggtgagtgctgttgttca-3' (NOX1); $5^{\prime}$-accacagtccatgccatcac- $3^{\prime}$ and $5^{\prime}$-tccaccaccctgttgctgta-3' (GAPDH), respectively.

\section{Electrophoretic mobility shift assays (EMSA)}

Biotinylated double-stranded oligonucleotides were synthesized by Bioneer Co. (Korea) as follows: NF-кB, 5'-agttgaggggactttcccaggc-3'; NF-Y, $5^{\prime}$-agaccgtacgtgattggttaatctctt-3'. Nuclear extracts were prepared from BMM cells stimulated with RANKL

Published by Bioscientifica Ltd. 
(40 ng/mL) using NE-PER nuclear and cytoplasmic extraction reagents (Pierce). Binding reactions were carried out for $20 \mathrm{~min}$ at room temperature in the presence of $50 \mathrm{ng} / \mathrm{mL}$ poly(dI-dC), 0.05\% Nonidet P-40, $5 \mathrm{mM} \mathrm{MgCl}_{2}$, $10 \mathrm{mM}$ EDTA and $2.5 \%$ glycerol in $1 \times$ binding buffer using $20 \mathrm{fM}$ of biotin-end-labeled target DNA and $3 \mu \mathrm{g}$ of nuclear (LightShift Chemiluminescent EMSA kit; Pierce). Samples were loaded onto native $6 \%$ polyacrylamide gels pre-electrophoresed for $60 \mathrm{~min}$ in $0.5 \times$ Tris borate/EDTA and electrophoresed at $100 \mathrm{~V}$ before being transferred onto positively charged nylon membranes (Hybond-N+) in $0.5 \times$ Tris borate/EDTA at $100 \mathrm{~V}$ for $30 \mathrm{~min}$. Transferred DNAs were cross-linked to the membrane at $10 \mathrm{~mJ} / \mathrm{cm}^{2}$ and detected using horse radish peroxidase (HRP)-conjugated streptavidin.

\section{Plasmid construction}

The NOX1 gene was V5-tagged by subcloning into the pcDNA6-V5-His-A vector (Invitrogen) containing a C-terminal V5 tag between NheI and NotI. The FLAGtagged Nod2 gene was subcloned into pcDNA3.1 vector (Addgene, MA, USA) between BamHI and EcoRI. All constructs were sequenced to verify $100 \%$ agreement with the original sequence.

\section{Co-immunoprecipitation and Western blots}

HEK293T cells $\left(8 \times 10^{6}\right)$ grown in Dulbecco's modified Eagle's medium supplemented with $10 \%$ fetal bovine serum were transfected with $5 \mu$ g samples of the expression vectors using Lipofectamine 3000 (Invitrogen). After 48h, cells were harvested and lysed by incubating with RIPA buffer $(50 \mathrm{mM}$ Tris- $\mathrm{HCl} \mathrm{pH} 7.5,1 \%$ Triton-X 100, 0.5\% sodium deoxycholate, $150 \mathrm{mM} \mathrm{NaCl}, 1 \mathrm{mM}$ EDTA, $1 \%$ protease inhibitor mixture) for $20 \mathrm{~min}$. Cell pellets were removed by centrifugation at $16,500 \boldsymbol{g}$ for $10 \mathrm{~min}$. The lysates were incubated with Anti-V5-tag mAb-Magnetic beads (MBL, Nagoya, Japan) $\mathrm{O} / \mathrm{N}$ with rotation at $4^{\circ} \mathrm{C}$. Immunoprecipitates were then washed three times with RIPA buffer and eluted with SDS sample buffer by incubation at $100^{\circ} \mathrm{C}$ for $2 \mathrm{~min}$. Proteins were separated by SDS-PAGE, and western blotting was performed using monoclonal HRP-conjugated anti-FLAG M2 (Sigma).

\section{Measurement of intracellular reactive oxygen species}

Intracellular ROS were detected using the fluorescent probe 2',7'-dichlorofluorescein diacetate ( $\left.\mathrm{H}_{2} \mathrm{DCFDA}\right)$
(Molecular Probes). BMMs were cultured under various experimental conditions for $48 \mathrm{~h}$, harvested, suspended in PBS, loaded with $\mathrm{H}_{2}$ DCFDA and incubated at $37^{\circ} \mathrm{C}$ for $30 \mathrm{~min}$. Intracellular ROS were measured by flow cytometry with a FACSCalibur.

\section{Statistical analysis}

Values are expressed as means \pm standard error of mean (s.E.M.). Student's $t$-test was used to evaluate the differences between samples and corresponding controls. Differences between groups were assessed by one-way ANOVA, followed by Bonferroni posttests. A $P$ value of less than 0.05 was considered statistically significant.

\section{Results}

\section{NOD2 deficiency increases bone density upon OVX-induced bone loss}

We hypothesized that NOD2 reduces bone mass by acting on OCs, since it interacts with serine-threonine kinases to induce the activation of NF-кB (Ogura et al. 2001), which is important for osteoclastogenesis (Iotsova et al. 1997). To test this hypothesis, we set up a bone loss model involving OVX in mice and compared the femurs of Nod2-KO mice and their littermates by $\mu \mathrm{CT}$. The absence of NOD2 attenuated the bone loss induced by OVX and had no significant effect on sham-operated mice (Fig. 1A). It also increased bone mineral density (BMD), bone volume (BV/TV), trabecular number (Tb. N.) and trabecular thickness (Tb. Th.) and reduced the enlargement of trabecular space (Tb. Sp.) (Fig. 1B). As shown in Fig. 1C, OC.N/BS (OC number over total bone surface) in in vivo TRAP staining was also reduced in the absence of NOD2 upon OVX (Fig. 1C). Consistent with this, OC formation increased significantly in ex vivo cultures of BMM-enriched populations derived from WT OVX mice, while there were fewer OC in parallel cultures from Nod2-KO OVX mice (Fig. 1D). NOD2 deficiency also significantly reduced the large OCs that were evaluated by the area of OCs compared to sham-operated WT. Ex vivo cultures of whole bone marrow cells showed a similar trend to that of enriched BMMs in the absence of NOD2 (Fig. 1E). Consistent with this, serum CTX-1, an in vivo bone resorption marker, was also reduced in the absence of NOD2 upon OVX (Table 1). OVX also increased levels of the in vivo bone formation markers serum ALP and osteocalcin in WT mice, but lack of NOD2

Published by Bioscientifica Ltd 
A
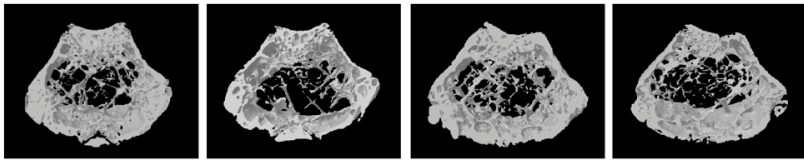

SHAM

OVX

WT

SHAM

OVX

B
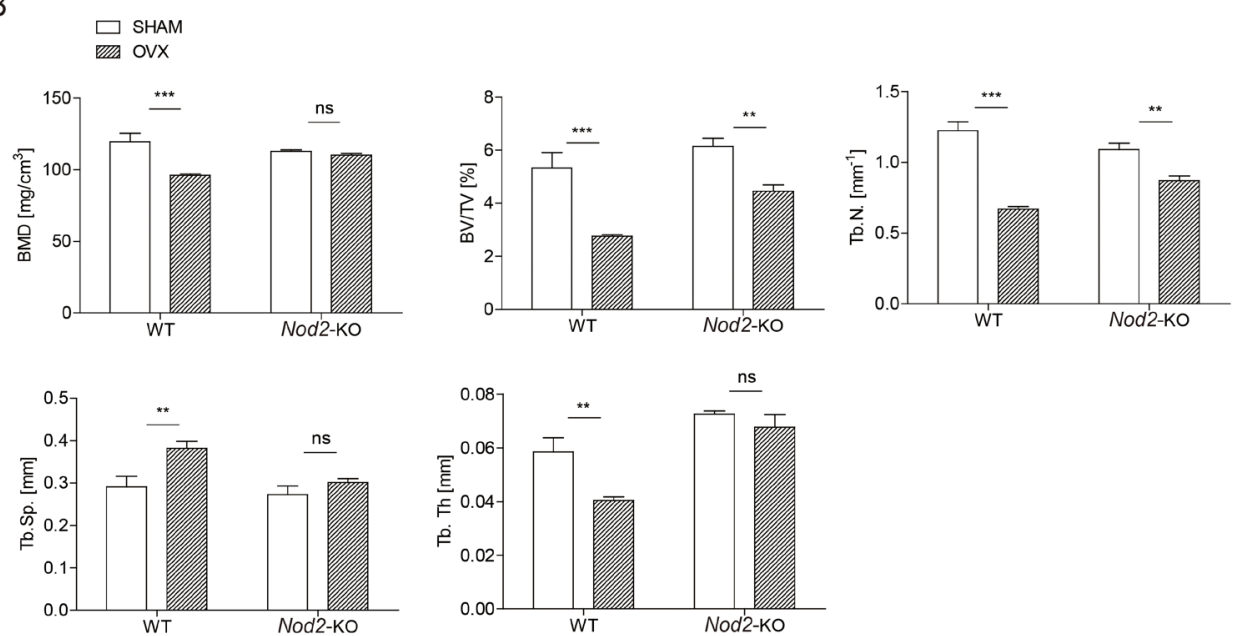

C
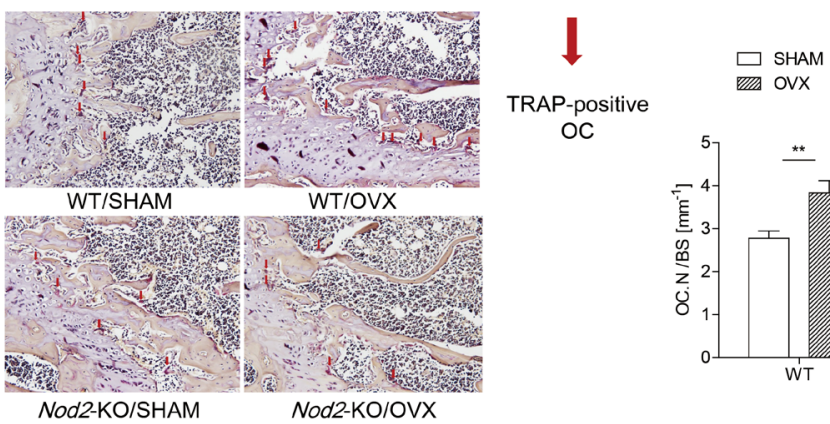

TRAP-positive

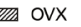

OC

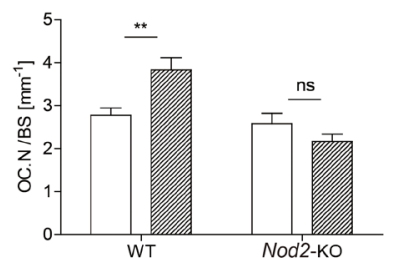

D
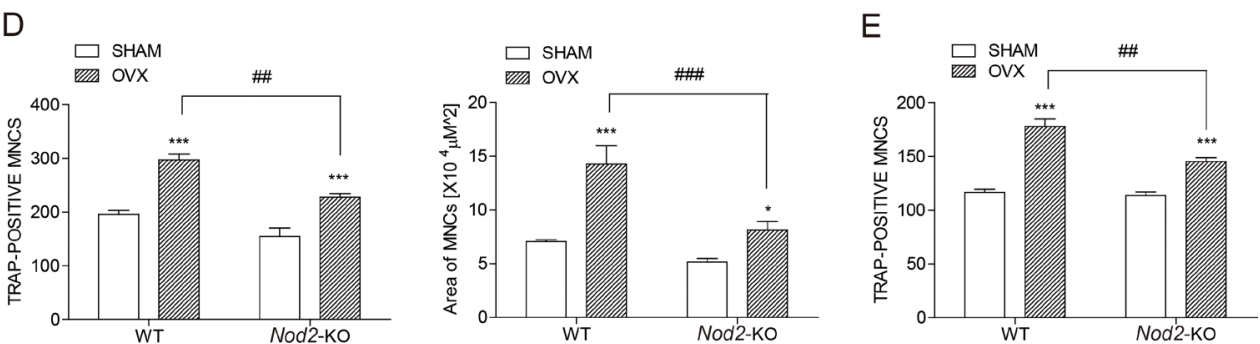

Figure 1

Absence of NOD2 protects against bone loss induced by OVX in mice. Representative $\mu C T$ images of distal mouse femora of WT mice $1.0 \mathrm{~mm}$ from the growth plate (OVX, $n=6$; sham, $n=6)$ and Nod2-KO mice (OVX, $n=6$; sham, $n=6)$ eight weeks after OVX and sham surgery (A). Trabecular BMD, trabecular BV/TV, Tb. N., Tb. Th. and Tb. Sp. were measured by $\mu \mathrm{CT}$ (B). To examine in vivo TRAP-positive OCs, mouse femora were excised, cleaned with a soft tissue and decalcified in EDTA. Representative histological sections of the distal femoral metaphysis of WT and Nod2-KO mice were stained for TRAP to identify OCs (original magnification $\times 200$ ) with OC.N/BS (OC number over total bone surface) (C). Enriched BMMs were stimulated with RANKL $(40 \mathrm{ng} / \mathrm{mL}) / \mathrm{M}$-CSF $(20 \mathrm{ng} / \mathrm{mL})$ for 3 days, and TRAP-positive MNCs per well were counted after fixation. Thereafter, more than 70 TRAP-positive MNCs in each culture were randomly selected, and the area of the formed OCs were measured (D). Whole bone marrow cells were stimulated with $1,25(\mathrm{OH})_{2} \mathrm{D}_{3}$ $(10 \mathrm{nM})$ and $\mathrm{PGE}_{2}(1 \mu \mathrm{M})$ for 7 days $(\mathrm{E})$. Data are expressed as means \pm S.E.M. ${ }^{*} P<0.05 ; * * P<0.01 ; * * * P<0.001$ compared with corresponding SHAM.

Differences between groups were analyzed by two-way ANOVA, followed by Bonferroni posttests to compare the effect of genotype (Tb. Sp.; $P<0.05$, BV/TV; $P<0.01$, Tb. Th. and OC. N/BS; $P<0.001$ ), the effect of surgery (Tb. Sp., Tb. Th.; $P<0.01$, BMD, BV/TV, and Tb. N.; $P<0.001)$ and interaction (BMD and OC.N/BS; $P<0.01$, Tb. N.; $P<0.001$ ). Similar results were obtained in three independent experiments.

http://joe.endocrinology-journals.org DOI: $10.1530 / \mathrm{JOE}-16-0591$
() 2017 Society for Endocrinology Printed in Great Britain
Published by Bioscientifica Ltd 
Table 1 Biochemical markers of OVX and SHAM mice in WT and Nod2-KO mice at 8 weeks after surgery.

\begin{tabular}{|c|c|c|c|c|}
\hline & \multicolumn{2}{|c|}{ WT } & \multicolumn{2}{|c|}{ Nod2-KO } \\
\hline & SHAM & ovx & SHAM & ovx \\
\hline CTX (ng/mL) & $17.72 \pm 1.188$ & $30.73 \pm 1.853 * * *$ & $14.89 \pm 1.124$ & $24.16 \pm 1.050 * * *$ \\
\hline ALP (U/L) & $35.29 \pm 1.606$ & $53.24 \pm 4.223 * *$ & $40.66 \pm 1.931$ & $42.81 \pm 4.049$ \\
\hline OCN (ng/mL) & $21.76 \pm 2.450$ & $42.01 \pm 1.450 * * *$ & $22.65 \pm 1.682$ & $36.14 \pm 1.723 * * *$ \\
\hline
\end{tabular}

Data are represented as mean \pm S.E.M. Differences between groups were analyzed by two-way ANOVA, followed by Bonferroni posttests to compare the effect of genotype $(C T X, P<0.01)$ and the effect of surgery (ALP, $P<0.01 ; C T X$ and $O C N, P<0.001)$, and interaction between genotype and surgery (ALP, $P<0.05)$. Similar results were obtained in three independent experiments. ${ }^{*} P<0.01 ; * * *<0.001$ compared with SHAM.

did not reduce these levels. NOD2 deficiency also did not significantly affect serum ALP and osteocalcin after sham surgery (Table 1).

\section{NOD2 deficiency decreases osteoclastogenesis in vitro}

To further examine the role of NOD2 in OCs, Nod2 expression was evaluated upon RANKL stimulation. Stimulation of BMMs with RANKL to induce their differentiation into OCs appeared to slightly increase transcripts of Nod2, but the effect was not significant (Fig. 2A). In addition, Nod2 mRNA levels in OC were not significantly changed upon OVX (Fig. 2A). To see whether NOD2 affected osteoclastogenesis in vitro, we examined OC formation in cultures of BMMs from WT and Nod2-KO mice, the BMMs were free of stromal cells and lymphocytes. The two factors necessary for OC differentiation, M-CSF and RANKL, induced maximal OC formation after 3-days exposure, and OC formation in the Nod2-KO BMMs was 28\% lower than that in the WT BMMs, as measured by counting TRAP-positive MNCs (Fig. 2B and C). NOD2 deficiency reduced the area of OCs with a greater extent (45\% reduction) than the number of OCs (Fig. 2C). Since the area of OCs was more prominent than the number of OCs in the absence of NOD2, we used the area of OCs to evaluate the effect of NOD2 in further studies. To confirm the effects of NOD2, we used MDP to stimulate endogenous NOD2 in WT cells. MDP,
A

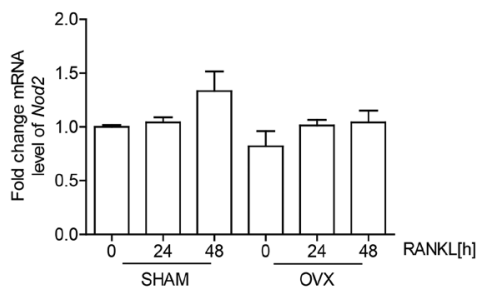

B

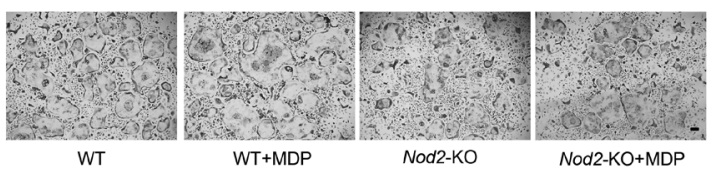

C
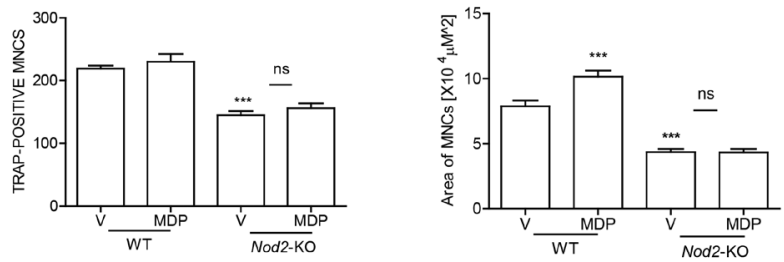

D
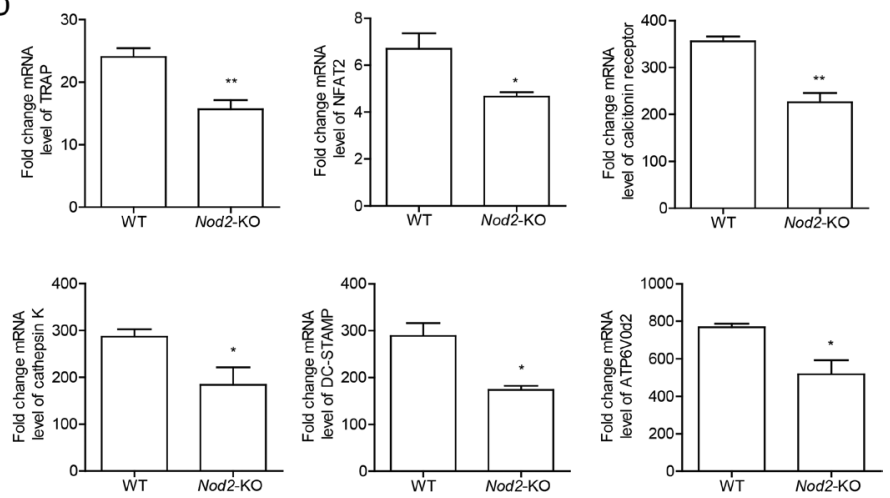

Figure 2

NOD2 deficiency decreases the formation of OCs. RNA from BMMs stimulated with RANKL (40 ng/mL) and M-CSF (20 ng/mL) at indicated time points was measured by qPCR for Nod2. Expression before RANKL treatment was set at 1 (A). BMMs from WT and Nod2-KO mice were incubated in the presence of M-CSF and stimulated with RANKL plus or minus MDP $(10 \mu \mathrm{g} / \mathrm{mL}$, Sigma Chemical) (B, C). Scale bar; $100 \mu \mathrm{m}$ in representative photos of OCs (B). Cells were fixed after 3 days, more than 70 TRAP-positive MNCs in each culture were randomly selected, and the area of the formed OCs was measured. RNA from BMMs stimulated with RANKL and M-CSF at $48 \mathrm{~h}$ was measured by qPCR for OC-specific genes (D) $* P<0.05 ; * * P<0.01 ; * * * P<0.001$ compared with WT cells. Similar results were obtained in three independent experiments.

http://joe.endocrinology-journals.org DOI: 10.1530/JOE-16-0591
() 2017 Society for Endocrinology Printed in Great Britain
Published by Bioscientifica Ltd 
a ligand of NOD2, did not increase the number of OC, but the area of OCs was increased. No further changes with MDP were found in the absence of NOD2 (Fig. 2C). As shown in Fig. 2D, transcript levels of TRAP, cathepsin $\mathrm{K}$, calcitonin receptor, NFAT2, ATP6v0d2 and DC-STAMP were significantly reduced in response to RANKL in the OCs from the Nod2-KO mice.

\section{NOD2 deficiency decreases OC function in vitro}

To examine whether NOD2 affects OC activity, we examined bone resorption in vitro using dentine slices. Mature OCs generated from the cells transfected with siNOD2 gave rise to a significantly lower total pit area/ number of OC compared to those from the cells transfected with scRNA (Fig. 3A). Stimulation of endogenous NOD2 by MDP in mature OCs increased total pit area/number of
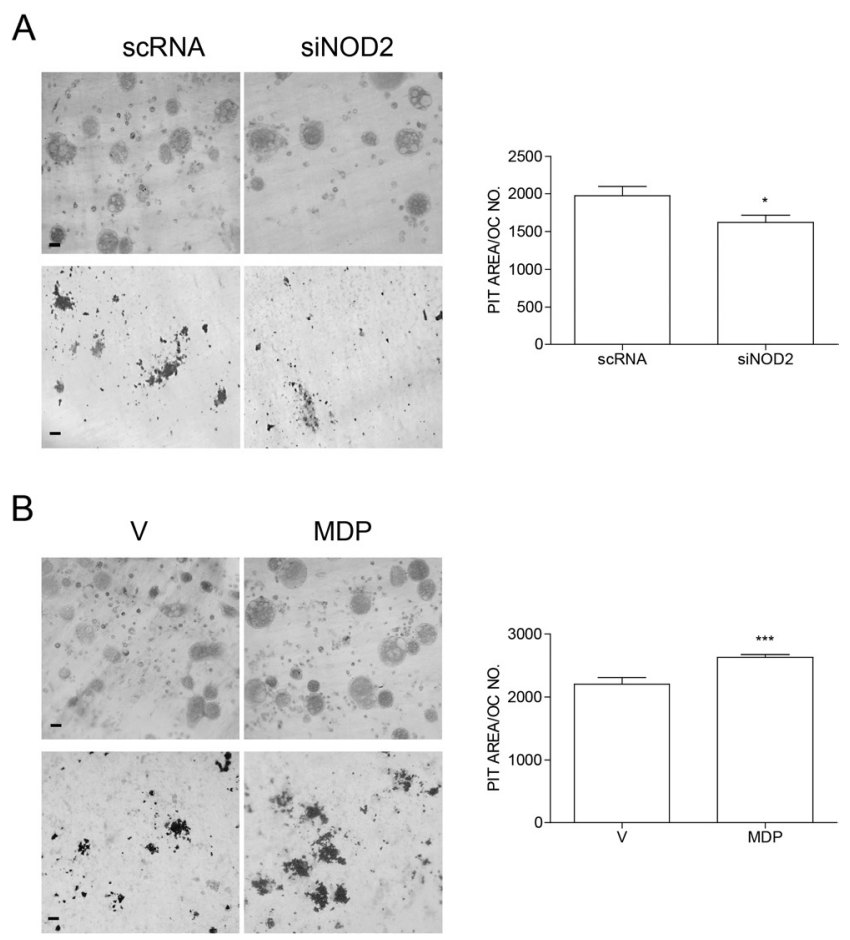

Figure 3

NOD2 deficiency decreases OC activity. BMMs were transfected with siNOD2 or scRNA for $8 \mathrm{~h}$, and then cultured with M-CSF $(20 \mathrm{ng} / \mathrm{mL})$ and RANKL $(40 \mathrm{ng} / \mathrm{mL})$ on whole dentine slices to generate mature OCs for 7 days (A). Mature OCs were generated on whole dentine slices with M-CSF and RANKL with or without MDP $(10 \mu \mathrm{g} / \mathrm{mL})$ for 6 days (B). Cells were fixed with formalin and stained for TRAP. Then, cells were removed and the slices were stained with Mayer's hematoxylin. Representative photos of TRAP-positive OCs and resorption pits formed are shown. Scale bar: $50 \mu \mathrm{m}$. Total pit area/number of TRAP-positive OC was measured. $* P<0.05 ; * * * P<0.001$ compared with vehicle-treated or scRNAtransfected cells. Similar results were obtained in three independent experiments.
OC significantly (Fig. 3B), suggesting that stimulation of NOD2 augments the resorptive capacity of mature OCs.

\section{NOD2 affects RANKL signaling by activating NF- $\kappa$ B during osteoclastogenesis}

To gain insight into how NOD2 elevates the number and activity of OCs, we investigated the effect of NOD2 deficiency on RANKL-induced signaling. Cross-linking between RANK and RANKL activates the key transcription factor, NF-kB (Iotsova et al. 1997), resulting in the expression of OC-specific genes. We asked whether NOD2 affected the activation of NF-кB. As shown in Fig. 4A, stimulation of WT BMMs with RANKL for $1 \mathrm{~h}$ induced NF-kB DNA-binding activity and stronger NF-кB DNA binding was observed after treatment with MDP. Similar pattern was observed with 2-h stimulation. NF-kB DNAbinding activity was lower in the absence of NOD2, and there was no further change in response to MDP. The specificity of the binding activity was confirmed by competition assays using excess unlabeled probe. To clarify the mode of action of NOD2 in NF- $\mathrm{kB}$ activation, we compared the effects of a pharmacological inhibitor of the nuclear translocation of $\mathrm{p} 65$ with those of an inhibitor of I $\mathrm{B} \alpha \alpha$ phosphorylation on OC formation in response to NOD2 activation by MDP. Both inhibitors decreased the area of OC significantly and abolished the increase induced by MDP (Fig. 4B). A similar pattern was observed with the expression of OC-specific genes including TRAP, calcitonin receptor and ATP6v0d2, supporting the morphological evaluation of OCs. Next, knockdown of Nod2 by siNOD2 reduced OC-specific genes as well as the area of OCs. Those reductions were attenuated by blockade of NF-кB activation (Fig. 4C), confirming that NOD2 plays a role via NF-kB activation.

\section{NOD2 deficiency decreases RANKL-induced ROS}

Since our previous finding showed OVX-induced oxidative stress (Van Phan et al. 2013), we tested whether NOD2 was implicated in the induction of oxidative stress. The absence of NOD2 decreased the elevation of serum ROS in response to OVX (Fig. 5A). Then, we examined whether NOD2 affected the sustained level of ROS in OCs stimulated by RANKL, since ROS plays an important role in RANKL signaling during OC formation (Lee et al. 2005). Treatment with RANKL induced a sustained level of ROS over $48 \mathrm{~h}$ of exposure, but NOD2 deficiency reduced the RANKL-induced ROS level significantly

Published by Bioscientifica Ltd. 
A
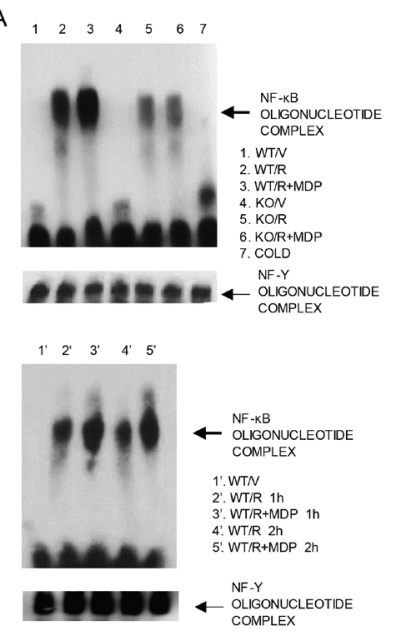

B
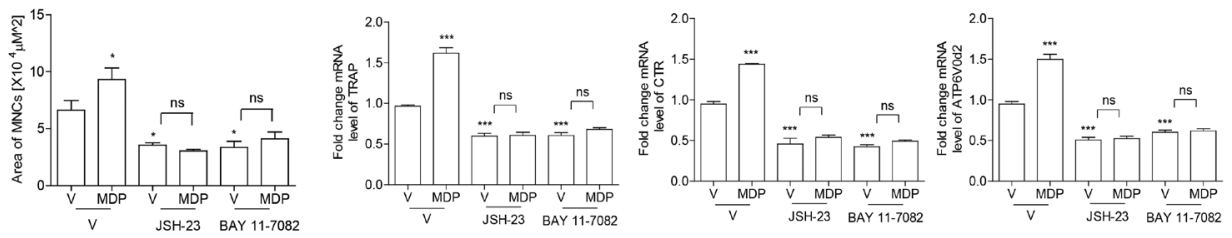

C
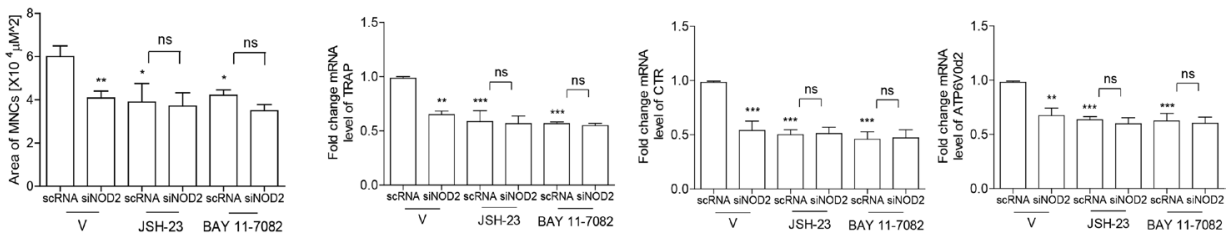

Figure 4

Attenuated RANKL signaling in OCs in the absence of NOD2. BMMs from WT and Nod2-KO mice were stimulated with vehicle (lane 1, 4), RANKL (100 ng/ $\mathrm{mL})($ lane 2,5$)$ or RANKL along with MDP $(10 \mu \mathrm{g} / \mathrm{mL})($ lane 3,6$)$ for $1 \mathrm{~h}$. A 100 -fold excess of unlabeled probe (lane 7$)$ was added as a negative control (Left panel). BMMs from WT mice were stimulated with vehicle (lane 1'), RANKL (100 ng/mL) (lane 2', 4') or RANKL along with MDP (10 $\mu$ g/mL) (lane 3', $\left.5^{\prime}\right)$ for $1 \mathrm{~h}\left(1^{\prime}, 2^{\prime}, 3^{\prime}\right)$ and $2 \mathrm{~h}\left(4^{\prime}, 5^{\prime}\right)$ (Right panel). NF-Y DNA binding activity served as an internal control (A). BMMs were treated with BAY $11-7082$ $(5 \mu \mathrm{M})$ or JSH-23 $(10 \mu \mathrm{M})$, and stimulated with RANKL or RANKL + MDP $(10 \mu \mathrm{g} / \mathrm{mL})(\mathrm{B})$ for $48 \mathrm{~h}$ and $72 \mathrm{~h}$ to measure the expression of TRAP, calcitonin receptor, and ATP6v0d2 and the area of OCs, respectively. BMMs were transfected with siNOD2 or scRNA for $8 \mathrm{~h}$ before receiving above treatment (C). ${ }^{*} P<0.05 ; * * P<0.01 ; * * * P<0.001$ compared with RANKL-treated cells receiving vehicle $(\mathrm{V})$ or scRNA-transfected RANKL-treated cells. Similar results were obtained in three independent experiments.

(Fig. 5B). The sustained level of ROS is the net outcome of production and removal of ROS; to evaluate whether NOD2 affected the production or the removal of ROS, we determined RANKL-induced ROS levels in the presence of N-acetylcysteine (NAC), a general antioxidant, and diphenyliodonium (DPI), a selective inhibitor of NOX, in the absence of NOD2. A further decrease of ROS level in the absence of NOD2 was seen in the presence of NAC, but not of DPI (Fig. 5C), suggesting that the NOD2induced increase in ROS was due to increased production of ROS rather than reduced removal of ROS. To confirm that NOD2 was associated with RANKL-induced ROS production, we knocked down Nox1 by transfection with siNOX1. As shown in Fig. 5D, downregulation of the RANKL-specific NADPH oxidase by siNOX1 decreased ROS, but the extent of the reduction was not affected by the absence of NOD2. A similar pattern was observed when the area of OCs was measured (Fig. 5E).

Since oxidative stress induced by OVX was attenuated in the absence of NOD2, we wondered whether NOD2 was implicated in the effects of estrogen $\left(\mathrm{E}_{2}\right)$ on ROS levels. As shown in Fig. 5F, $\mathrm{E}_{2}$ reduced RANKL-induced ROS levels in WT cells, but not in Nod2-KO cells, suggesting that NOD2 is associated with the $\mathrm{E}_{2}$ effect in modulating ROS levels in OCs. A similar pattern was found for decreasing the area of OCs in response to $\mathrm{E}_{2}$ (Fig. $5 \mathrm{G}$ ).
Since there are reports of a direct interaction between TLR components and NOX family members (Lipinski 2009, Park 2004), we examined whether NOD2 physically interacted with NOX1 in OCs. Co-expression of FLAGtagged NOD2 with V5-tagged NOX1 in HEK293 cells was analyzed by co-immunoprecipitation. As shown in Fig. 6, precipitation of overexpressed V5-tagged NOX1 resulted in co-precipitation of FLAG-tagged NOD2, supporting a direct physical interaction between NOX1 and NOD2.

\section{Discussion}

We have shown that absence of NOD2 reduces the bone loss induced by OVX in mice. OVX has been shown to induce a modest amount of bone loss when 5- to 6-weekold mice (Lee et al. 2006a), 8-week-old mice (Lee et al. $2006 b$ ) and 16-week-old mice (Gao et al. 2007) were subjected to OVX and analyzed 4 weeks later. OVX induced an immediate decrease of bone formation after 2 weeks ( $\mathrm{Li}$ et al. 2011), but thereafter, there was a sustained increase (Cenci et al. 2000). In the present work, we carried out OVX on 6-week-old Nod2-KO mice and analyzed its effect 8 weeks later. The OVX induced significant bone loss in WT mice, but not in Nod2-KO mice. As all the mice used were not raised under germ-free condition, but under 
A

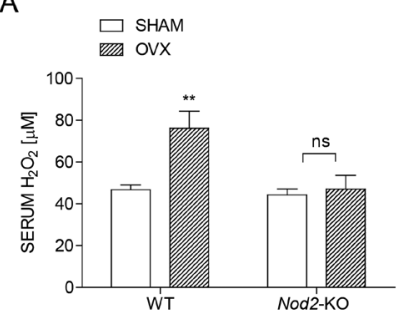

B

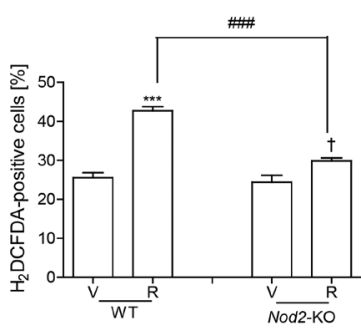

C

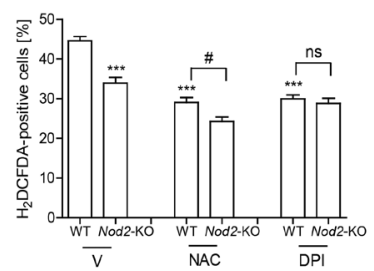

E

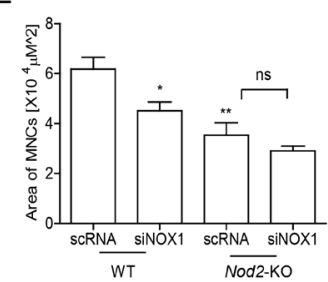

$\mathrm{F}$

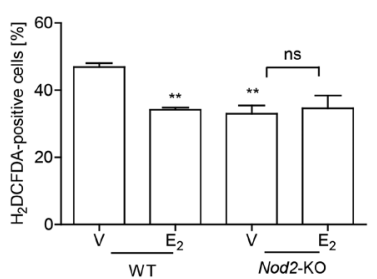

G

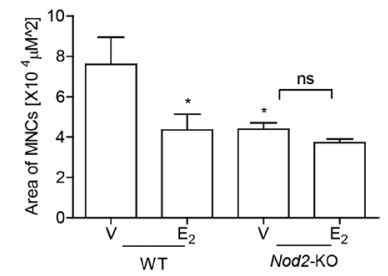

Figure $\mathbf{5}$

NOD2 deficiency decreases RANKL-induced ROS. Serum ROS was determined from OVX and SHAM mice in WT and Nod2-KO mice at 8 weeks after surgery (A). Differences between groups were analyzed by two-way ANOVA, followed by Bonferroni posttests to compare the effect of genotype $(P<0.05)$ and the effect of surgery $(P<0.01)$, and interaction between genotype and surgery $(P<0.05)$. BMMs from WT and Nod2-KO mice were stimulated with RANKL along with M-CSF (B) and treated with NAC (3 mM), DPI (5nM) (C); or $E_{2}(10 \mathrm{nM})(\mathrm{F}, \mathrm{G})$. For $\mathrm{E}_{2}$ treatment, BMMs were cultured in $\alpha$-MEM without phenol red containing $10 \%$ charcoal-treated FBS. Cultures were incubated for $48 \mathrm{~h}$ to determine intracellular levels of ROS by flow cytometry using $\mathrm{H}_{2} \mathrm{DCF}-\mathrm{DA}(\mathrm{B}, \mathrm{C}, \mathrm{D}, \mathrm{F})$ and for $72 \mathrm{~h}$ to determine the area of OCs $(\mathrm{E}, \mathrm{G}) .{ }^{*} P<0.05 ;{ }^{*} P<0.01 ; * * * P<0.001$ compared with WT cells. ${ }^{\dagger} P<0.05$ compared with Nod2-KO cells. $\# P<0.05 ; \# \# P<0.001$ compared with the corresponding WT cells. BMMs were transfected with siNOX1 or scRNA. Downregulation of Nox 1 by siRNA was confirmed by RT-PCR and qPCR. After $8 \mathrm{~h}$, cells were stimulated with RANKL to determine intracellular ROS using $\mathrm{H}_{2}$ DCF-DA for $48 \mathrm{~h}(\mathrm{D})$ and to determine the area of OCs for $72 \mathrm{~h}(\mathrm{E})$, respectively. ${ }^{*} P<0.05 ; * * P<0.01$; ${ }^{* *} P<0.001$ compared with RANKL-treated scRNA-transfected cells (V). Similar results were obtained in three independent experiments.

specific pathogen-free condition, microbiota could help MDP to activate NOD2 in WT mice. We focused on the trabecular microarchitecture of the ovariectomized mice, since postmenopausal women have been reported to undergo trabecular bone loss induced by elevated bone

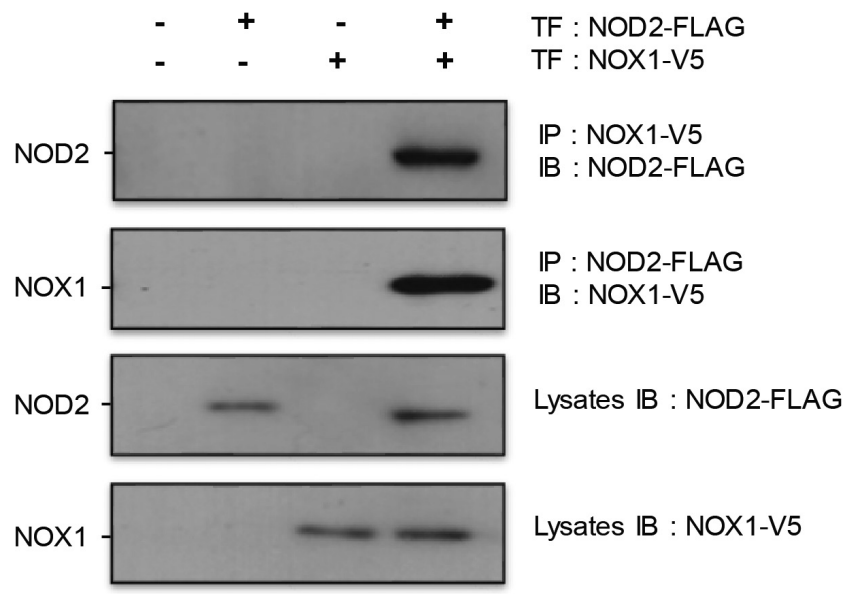

Figure 6

NOD2 interacts physically with NOX1. NOD2-FLAG and NOX1-V5 constructs were overexpressed in HEK293 cells. Immunoprecipitates and cell lysates were assayed for co-precipitated proteins as indicated. Similar results were obtained from three independent experiments. remodeling with excess bone resorption exceeding bone formation (Riggs \& Melton 1992, Recker et al. 2004). Nod2-KO OVX mice exhibited a significant increase in trabecular BMD, BV/TV, Tb. N. and Tb. Th. and a decrease of Tb. Sp. compared with their littermates. The absence of NOD2 reduced the number of OCs derived from BMMs in ex vivo cultures upon OVX as well as histological sections of femur from OVX mice, suggesting that the effect of NOD2 was through OCs. In accordance with this view, lack of NOD2 decreased the level of an in vivo marker of bone resorption, serum CTX-1, which is elevated upon OVX. However, no significant change in bone mass was observed in the absence of NOD2 after sham surgery. This discrepancy can be explained by stimulus-induced signal augmentation that are found in CD44 (Li et al. 2015) and leukotriene B4 receptor (Hikiji et al. 2009). It is likely that the protective effect of NOD2 deficiency upon OVX is due to preventing the increased activity of NOD2 in OCs that occurs in WT mice, hence implying that NOD2 is involved in the decrease of bone density upon OVX. At the same time, NOD2 deficiency did not increase bone formation, as indicated by serum ALP and osteocalcin levels, which implies that the observed bone phenotype of Nod2-KO mice is mainly due to decreased bone resorption rather

Published by Bioscientifica Ltd. 
than increased bone formation. Although lack of NOD2 did not alter bone formation in vivo, we cannot exclude a contribution of stromal cells/osteoblasts in the increased OC formation. In fact, increased osteoclastogenesis has been reported in response to MDP activation in osteoblasts (Yang et al. 2005).

The contribution of OCs to the attenuated bone loss in the absence of NOD2 is supported by our in vitro observation that, in the absence of NOD2, the number of OCs decreased as the result of decreased commitment to the OC lineage. Interestingly, the decrease of OC formation in the absence of NOD2 was more prominent in the area of OCs, but a decrease of ATP6v0d2 and DC-STAMP transcripts was similar to that of other OC-specific genes in the absence of NOD2, suggesting that NOD2 may not specifically participate in fusion. In addition, the activity of OCs was reduced in the absence of NOD2. These results suggested that NOD2 augments the resorptive capacity of OCs as well as enhances large OCs. Since our in vitro studies (except for Fig. 2A) were performed with cells from male mice to avoid periodic $\mathrm{E}_{2}$ effects, we examined the expression of RANKL-induced OC-specific genes in cells from female mice. The absence of NOD2 reduced the expression of these genes but to a lesser extent than that in the cells from male mice (Supplementary Fig. 1, see section on supplementary data given at the end of this article). This agrees with the finding that NOD2 deficiency had less effect on ex vivo OC formation in cells from female mice, as well as the finding that NOD2 deficiency abolished the effect of $\mathrm{E}_{2}$ on OC formation in $\mathrm{E}_{2}$-free conditions. Taken together, these results suggest that NOD2 is responsible, at least in part, for the increased OC formation upon OVX.

We found that Nod2 mRNA levels did not change upon OVX and that they increased only modestly, if at all, during OC formation, whereas others (Prates et al. 2014) have reported that the levels increased significantly, and that bacterial stimulation also elevated them (Liu et al. 2014). This discrepancy suggests Nod2 expression in OCs does not change very much. It is well known that NOD2 levels are regulated by posttranslational modification (Tigno-Aranjuez \& Abbott 2012), not by transcript levels. While we were investigating the effect of NOD2 in our OVX-induced bone loss model, Prates and coworkers (Prates et al. 2014) reported that lack of NOD2 led to lower bone resorption in an experimental model of periodontitis 4 weeks after Porphyromonas gingivalis ( $P$. gingivalis) infection. On the other hand, absence of NOD2 along with ApoE deficiency led to alveolar bone loss as well as atherosclerosis and an increase of inflammatory cytokines after 15 weeks of Porphyromonas gingivalis infections (Yuan et al. 2013). Dual deficiency of NOD2 and ApoE appears to exacerbate impairing lipoprotein profiles, exhibiting chronic inflammation. The discrepancy between two findings could be due to the differences of environment and/or duration of stress. It is likely that NOD2 may behave oppositely under different microenvironments upon same stress, although it is not clear yet to define the role of NOD2 in inflammatory bone loss.

We showed that NOD2 deficiency antagonized RANKL signaling by decreasing the area of OCs. In the absence of NOD2, NF-кB DNA binding activity was attenuated (Fig. 4A). The specific inhibitors of NF-kB, JSH-23 (inhibitor of p65 nuclear translocation) and BAY 11-7082 (IкB $\alpha$ phosphorylation inhibitor) abolished the effects of NOD2 on the area of OC during NOD2 activation and NOD2 deficiency (Fig. 4B and C), suggesting that NOD2 plays a critical role upstream of cytoplasmic phosphorylation in the NF- $\mathrm{kB}$ signaling pathway.

We also showed that NOD2 deficiency reduced OVXinduced oxidative stress. Our in vitro data demonstrated that NOD2 acted to increase the sustained ROS level induced by RANKL but not the level induced by M-CSF (Fig. 5B) and that inhibition of NOX or knockdown of Nox 1 abolished the decrease in ROS seen in the absence of NOD2 (Fig. 5D and E). It is well known that elevation of ROS upon RANKL stimulation contributes to OC formation (Lee et al. 2005). Our previous studies also showed that ROS participates in enhancing the number and activity of OCs by oxidation of c-Src and SHP-1 (Ke et al. 2014). The finding that NOD2 increased the differentiation and function of OCs (Figs 2 and 3), at least part, by increasing ROS is in agreement with findings that implicate other NLR family members in intracellular redox control. Thus, the assembly and activation of the NALP3 inflammasome is reported to be stimulated by ROS (Dostert et al. 2008), and NOD2-dependent ROS formation via dual oxidase 2 (DUOX2) has been shown to protect against bacterial infections (Lipinski et al. 2009). Binding of TLR4 to LPS stimulates NF-kB activation and ROS generation via NOX4 (Park et al. 2004). In addition, our data clearly show that NOD2 physically interacts with NOX1, thus paralleling the complex formation between NOX4 and TLR4 (Park et al. 2004) and between NOD2 and DUOX2 (Lipinski et al. 2009). However, it is possible that NOD2 interacts with NOX4, which regulates bone mass by generating hydrogen peroxide in OCs (Goettsch et al. 2013).

Taken together, we demonstrate that NOD2 deficiency attenuates the bone loss caused by OVX in mice. Our data also point to previously unrecognized effects of

Published by Bioscientifica Ltd. 
NOD2-dependent ROS formation on osteoclastogenesis. They indicate that NOD2 acts as a molecular switch that modulates differentiation and activity of OCs by increasing ROS generation in OCs. These results suggest NOD2 as a potential therapeutic target to reduce bone loss and oxidative stress due to loss of ovarian function.

\section{Supplementary data}

This is linked to the online version of the paper at http://dx.doi.org/10.1530/ JOE-16-0591.

\section{Declaration of interest}

The authors declare that they have no conflict of interest that could be perceived as prejudicing the impartiality of the research reported.

\section{Funding}

This work was supported by the Basic Science Research Program (2015R1A2A2A01002417) funded by the Korean government. K K (2014R1A1A2008740) and O J S (2014R1A6A1030318; 2016R1A6A3A11932375) were supported by the Basic Science Research Program through the National Research Foundation of Korea (NRF) funded by the Ministry of Education.

\section{Author contribution statement}

The study was designed by K K, O J S and H S C and was performed by K K, $\mathrm{O} J \mathrm{~S}$ and $\mathrm{S}$ W C. The manuscript was written by $\mathrm{K} \mathrm{K}$, O J S and $\mathrm{H} \mathrm{S} \mathrm{C}$ and revised by $\mathrm{KK}$, O J S and $\mathrm{H} \mathrm{SC}$.

\section{References}

Cappellen D, Luong-Nguyen N, Bongiovanni S, Grenet O, Wanke C \& Susa M 2002 Transcriptional program of mouse osteoclast differentiation governed by the macrophage colony stimulating factor and the ligand for the receptor activator of NF-кB. Journal of Biological Chemistry 277 21971-21982. (doi:10.1074/jbc.M200434200)

Cenci S, Weitzmann MN, Roggia C, Namba N, Novack D, Woodring J \& Pacifici R 2000 Estrogen deficiency induces bone loss by enhancing T-cell production of TNF-alpha. Journal of Clinical Investigation 106 1229-1237. (doi:10.1172/JCI11066)

Choi EK, Kim WK, Sul OJ, Park YK, Kim ES, Suh JH, Yu R \& Choi HS 2013 TNFRSF 14 deficiency protects against ovariectomy-induced adipose tissue inflammation. Journal of Endocrinology 220 25-33. (doi:10.1530/ JOE-13-0341)

Choi EK, Park HJ, Sul OJ, Rajasekaran M, Yu R \& Choi HS 2015 Carbon monoxide reverses adipose tissue inflammation and insulin resistance upon loss of ovarian function. American Journal of Physiology: Endocrinology and Metabolism 308 E621-E630. (doi:10.1152/ ajpendo.00458.2014)

Crotti TN, Dharmapatni AA, Alias E \& Haynes DR 2015 Osteoimmunology: major and costimulatory pathway expression associated with chronic inflammatory induced bone loss. Journal of Immunology Research 2015 281287. (doi:10.1155/2015/281287)
Dostert C, Pétrilli V, Van Bruggen R, Steele C, Mossman BT \& Tschopp J 2008 Innate immune activation through Nalp3 inflammasome sensing of asbestos and silica. Science 320 674-677. (doi:10.1126/ science.1156995)

de Faria AN, Zancanela DC, Ramos AP, Torqueti MR \& Ciancaglini P 2016 Estrogen and phenol red free medium for osteoblast culture: study of the mineralization ability. Cytotechnology 68 1623-1632. (doi:10.1007/ s10616-015-9844-2)

Eriksen EF, Langdahl B, Vesterby A, Rungby J \& Kassem M 1999 Hormone replacement therapy prevents osteoclastic hyperactivity: a histomorphometric study in early postmenopausal women. Journal of Bone and Mineral Research 14 1217-1221. (doi:10.1359/ jbmr.1999.14.7.1217)

Fuller K, Kirstein B \& Chambers TJ 2006 Murine osteoclast formation and function: differential regulation by humoral agents. Endocrinology 147 1979-1985. (doi:10.1210/en.2005-1340)

Gao Y, Grassi F, Ryan MR, Terauchi M, Page K, Yang X, Weitzmann MN \& Pacifici R 2007 IFN-gamma stimulates osteoclast formation and bone loss in vivo via antigen-driven $\mathrm{T}$ cell activation. Journal of Clinical Investigation 117 122-132. (doi:10.1172/JCI30074)

Ginaldi L \& De Martinis M 2016 Osteoimmunology and beyond. Current Medicinal Chemistry 23 3754-3774. (doi:10.2174/0929867323666160 907162546)

Girardin SE, Boneca IG, Viala J, Chamaillard M, Labigne A, Thomas G, Philpott DJ \& Sansonetti PJ 2003 Nod2 is a general sensor of peptidoglycan through muramyl dipeptide (MDP) detection. Journal of Biological Chemistry 278 8869-8872. (doi:10.1074/jbc.C200651200)

Goettsch C, Babelova A, Trummer O, Erben RG, Rauner M, Rammelt S, Weissmann N, Weinberger V, Benkhoff S, Kampschulte M, et al. 2013 NADPH oxidase 4 limits bone mass by promoting osteoclastogenesis. Journal of Clinical Investigation 123 4731-4718. (doi:10.1172/ JCI67603)

Hikiji H, Ishii S, Yokomizo T, Takato T \& Shimizu T 2009 A distinctive role of the leukotriene B4 receptor BLT1 in osteoclastic activity during bone loss. PNAS 106 21294-21299. (doi:10.1073/pnas.0905209106)

Hugot JP, Chamaillard M, Zouali H, Lesage S, Cézard JP, Belaiche J, Almer S, Tysk C, O'Morain CA, Gassull M, et al. 2001 Association of NOD2 leucine-rich repeat variants with susceptibility to Crohn's disease. Nature 411 599-603. (doi:10.1038/35079107)

Hughes DE, Dai A, Tiffee JC, Li HH, Mundy GR \& Boyce BF 1996 Estrogen promotes apoptosis of murine osteoclasts mediated by TGF-beta. Nature Medicine 2 1132-1136. (doi:10.1038/nm1096-1132)

Iotsova V, Caamaño J, Loy J, Yang Y, Lewin A \& Bravo R 1997 Osteopetrosis in mice lacking NF-kappaB1 and NF-kappaB2. Nature Medicine 3 1285-1289. (doi:10.1038/nm1197-1285)

Jimi E, Akiyama S, Tsurukai T, Okahashi N, Kobayashi K, Udagawa N, Nishihara T, Takahashi N \& Suda T 1999 Osteoclast differentiation factor acts as a multifunctional regulator in murine osteoclast differentiation and function. Journal of Immunology 163 434-442.

Ke K, Sul OJ, Choi EK, Safdar AM, Kim ES \& Choi HS 2014 Reactive oxygen species induce the association of SHP-1 with c-Src and the oxidation of both to enhance osteoclast survival. American Journal of Physiology: Endocrinology and Metabolism 307 E61-E70. (doi:10.1152/ ajpendo.00044.2014)

Ke K, Safder MA, Sul OJ, Kim WK, Suh JH, Joe Y, Chung HT \& Choi HS 2015 Hemeoxygenase-1 maintains bone mass via attenuating a redox imbalance in osteoclast. Molecular and Cellular Endocrinology 409 11-20. (doi:10.1016/j.mce.2015.03.022)

Kim WK, Choi EK, Sul OJ, Park YK, Kim ES, Suh JH \& Choi HS 2013 Monocyte chemoattractant protein-1 deficiency attenuates oxidative stress and protects against ovariectomy-induced chronic inflammation in mice. PLOS ONE 8 e72108. (doi:10.1371/journal. pone.0072108)

Kong YY, Yoshida H, Sarosi I, Tan HL, Timms E, Capparelli C, Morony S, Oliveira-dos-Santos AJ, Van G, Itie A, et al. 1999 OPGL is a key http://joe.endocrinology-journals.org

DOI: 10.1530/JOE-16-0591
๑) 2017 Society for Endocrinology Printed in Great Britain 
regulator of osteoclastogenesis, lymphocyte development and lymphnode organogenesis. Nature 397 315-323. (doi:10.1038/16852)

Lee NK, Choi YG, Baik JY, Han SY, Jeong DW, Bae YS, Kim N \& Lee SY 2005 A crucial role for reactive oxygen species in RANKL-induced osteoclast differentiation. Blood 105 852-859. (doi:10.1182/blood2004-09-3662)

Lee SKa, Kadono Y, Okada F, Jacquin C, Koczon-Jaremko B, Gronowicz G, Adams DJ, Aguila HL, Choi Y, Lorenzo JA, et al. 2006a T lymphocytedeficient mice lose trabecular bone mass with ovariectomy. Journal of Bone and Mineral Research 21 1704-1712. (doi:10.1359/jbmr.060726)

Lee SKb, Kalinowski JF, Jacquin C, Adams DJ, Gronowicz G \& Lorenzo JA $2006 b$ Interleukin-7 influences osteoclast function in vivo but is not a critical factor in ovariectomy-induced bone loss. Journal of Bone and Mineral Research 21 695-702. (doi:10.1359/jbmr.060117)

Li JY, Tawfeek H, Bedi B, Yang X, Adams J, Gao KY, Zayzafoon M, Weitzmann MN \& Pacifici R 2011 Ovariectomy disregulates osteoblast and osteoclast formation through the T-cell receptor CD40 ligand. PNAS 108 768-773. (doi:10.1073/pnas.1013492108)

Li Y, Zhong G, Sun W, Zhao C, Zhang P, Song J, Zhao D1, Jin X, Li Q, Ling S, et al. 2015 CD44 deficiency inhibits unloading-induced cortical bone loss through downregulation of osteoclast activity. Scientific Reports 5 16124. (doi:10.1038/srep16124)

Lipinski S, Till A, Sina C, Arlt A, Grasberger H, Schreiber S \& Rosenstiel P 2009 DUOX2-derived reactive oxygen species are effectors of NOD2-mediated antibacterial responses. Journal of Cell Science 122 3522-3530. (doi:10.1242/jcs.050690)

Liu J, Duan J, Wang Y \& Ouyang X 2014 Intracellular adhesion molecule-1 is regulated by porphyromonas gingivalis through nucleotide binding oligomerization domain-containing proteins 1 and 2 molecules in periodontal fibroblasts. Journal of Periodontology $\mathbf{8 5}$ 358-368. (doi:10.1902/jop.2013.130152)

Ogura Y, Inohara N, Benito A, Chen FF, Yamaoka S \& Nunez G 2001 Nod2, a Nod1/Apaf-1 family member that is restricted to monocytes and activates NF-kappaB. Journal of Biological Chemistry 276 4812-4818. (doi:10.1074/jbc.M008072200)

Park HS, Jung HY, Park EY, Kim J, Lee WJ \& Bae YS 2004 Cutting edge: direct interaction of TLR 4 with $\mathrm{NAD}(\mathrm{P}) \mathrm{H}$ oxidase 4 isozyme is essential for lipopolysaccharide-induced production of reactive oxygen species and activation of NF-kappa B. Journal of Immunology 173 3589-3593. (doi:10.4049/jimmunol.173.6.3589)

Prates TP, Taira TM, Holanda MC, Bignardi LA, Salvador SL, Zamboni DS, Cunha FQ \& Fukada SY 2014 NOD2 contributes to Porphyromonas gingivalis-induced bone resorption. Journal of Dental Research 93 1155-1162. (doi:10.1177/0022034514551770)

Recker R, Lappe J, Davies KM \& Heaney R 2004 Bone remodeling increases substantially in the years after menopause and remains increased in older osteoporosis patients. Journal of Bone and Mineral Research 19 1628-1633. (doi:10.1359/JBMR.040710)

Riggs BL \& Melton LJ 3rd 1992 The prevention and treatment of osteoporosis. New England Journal of Medicine 327 620-627. (doi:10.1056/NEJM199208273270908)

Rogers NH, Perfield JW, Strissel KJ, Obin MS \& Greenberg AS 2009 Reduced energy expenditure and increased inflammation are early events in the development of ovariectomy-induced obesity. Endocrinology 150 2161-2168. (doi:10.1210/en.2008-1405)

Rosenstiel P, Jacobs G, Till A \& Schreiber S 2008 NOD-like receptors: ancient sentinels of the innate immune system. Cellular and Molecular Life Sciences 65 1361-1377. (doi:10.1007/s00018-008-7502-y)

Suda T, Takahashi N, Udagawa N, Jimi E, Gillespie MT \& Martin TJ 1999 Modulation of osteoclast differentiation and function by the new members of the tumor necrosis factor receptor and ligand families. Endocrine Reviews 20 345-357. (doi:10.1210/edrv.20.3.0367)

Tigno-Aranjuez JT \& Abbott DW 2012 Ubiquitination and phosphorylation in the regulation of NOD2 signaling and NOD2mediated disease. Biochimica et Biophysica Acta 1823 2022-2028. (doi:10.1016/j.bbamcr.2012.03.017)

Van Phan T, Sul OJ, Ke K, Lee MH, Kim WK, Cho YS, Kim HJ, Kim SY, Chung HT, \& Choi HS 2013 Carbon monoxide protects against ovariectomyinduced bone loss by inhibiting osteoclastogenesis. Biochemical Pharmacology 85 1145-1152. (doi:10.1016/j.bcp.2013.01.014)

Weitzmann M \& Pacifici R 2006 Estrogen deficiency and bone loss: an inflammatory tale. Journal of Clinical Investigation 116 1186-1194. (doi:10.1172/JCI28550)

Yang S, Takahashi N, Yamashita T, Sato N, Takahashi M, Mogi M, Uematsu T, Kobayashi Y, Nakamichi Y, Takeda K, et al. 2005 Muramyl dipeptide enhances osteoclast formation induced by lipopolysaccharide, IL-1 alpha, and TNF-alpha through nucleotidebinding oligomerization domain 2-mediated signaling in osteoblasts. Journal of Immunology 175 1956-1964. (doi:10.4049/ jimmunol.175.3.1956)

Yuan H, Zelkha S, Burkatovskaya M, Gupte R, Leeman SE \& Amar S 2013 Pivotal role of NOD2 in inflammatory processes affecting atherosclerosis and periodontal bone loss. PNAS 110 E5059-E5068. (doi:10.1073/pnas.1320862110)

Received in final form 19 July 2017

Accepted 4 August 2017

Accepted Preprint published online 4 August 2017
๑) 2017 Society for Endocrinology Printed in Great Britain 